\title{
Ethical Principles of Virtual Leadership Construction in Artificial Intelligence Environment
}

\author{
Dong Pang ${ }^{1}$, Yushi Zhang ${ }^{2 *}$ \\ ${ }^{1}$ School of Management, Dalian Polytechnic University, Dalian, Liaoning,116034, China \\ ${ }^{2}$ School of Marxism, Liaoning Normal University, Dalian, Liaoning,116029, China
}

\begin{abstract}
In recent years, the discussion of artificial intelligence has become a trend. With artificial intelligence gradually replacing more human work, artificial intelligence plays the role of "semi rational man" in the era of big data. One of the key issues in the academic discussion is the ethical issues of artificial intelligence intervention in the work of rational social people, such as: Does the leadership of artificial intelligence intervention violate the ethical standards of most people? This paper takes the artificial intelligence intervention in the construction of virtual leadership as the thinking object, and puts forward some thoughts on the ethical principles of artificial intelligence.
\end{abstract}

\section{Introduction}

In the new stage of information development, big data has become a very important strategic resource. With the construction of big data system framework, the development of artificial intelligence has more "reference data". Therefore, we introduce a new field of thinking, that is, the value of service and decision-making in the artificial intelligence environment. This paper chooses the latter perspective of "decision value" to make an ethical perspective on the situation of AI intervention in Human resource management leadership. Artificial intelligence is an "irrational social person"[1]. As the object of scientific decision-making, if artificial intelligence, as a "semi rational person", enters the working group of all human beings, what kind of influence will it have? Today, with the development of science and technology, virtual teams are set up with the characteristics of cross time, space and region. For this kind of leadership decision, scholars such as Penny Pullan and Xiaobin Shen summarize it as "virtual leadership", which leads to the symmetry of information and leadership. The intervention of artificial intelligence has become a very effective means of subsidy. Of course, its intervention also brings some ethical conflicts between man and machine. This paper is based on this discussion.

\section{The construction of virtual leadership in the artificial intelligence environment}

From the essence of leadership, leadership is the unity of power, ability and influence. For the refinement of these three indicators, we reorganize two groups of indicators after investigating several leadership models, and compare them (Table 1). Among them, those marked with * are the functions that can be realized by artificial intelligence[2]. Therefore, in the artificial intelligence environment, the virtual leadership of an organization has its unique construction significance.

Table1. The most important thing for leaders is that they have or need to have such qualities

(Which red is the meeting point of the two sets of indicators)

\begin{tabular}{|l|l|}
\hline \multicolumn{1}{|c|}{ Indicator 1} & \multicolumn{1}{c|}{ Indicator 2} \\
\hline$\bullet$ Ability * & $\bullet$ Respect for others \\
\hline$\bullet$ Good judgment * & $\bullet$ Open to different views \\
\hline$\bullet$ Consistency & $\bullet$ Sincerity and transparency* \\
\hline$\bullet$ Vision & $\bullet$ Equality* \\
\hline$\bullet$ Self awareness & $\bullet$ Caring for others \\
\hline$\bullet$ Prudence ${ }^{*}$ & $\bullet$ Empathy \\
\hline$\bullet$ Sense of responsibility & $\bullet$ Confidence \\
\hline$\bullet$ Intelligence $*$ & $\bullet$ Keep the promise \\
\hline$\bullet$ Self control ${ }^{*}$ & $\bullet$ Listening \\
\hline$\bullet$ Empathy & $\bullet$ Organization* \\
\hline$\bullet$ Altruism & $\bullet$ Resilience* \\
\hline
\end{tabular}

\subsection{The background of virtual leadership in Al environment}

Virtual leadership is based on network virtual organization. There is no clear definition. According to the research, this paper defines virtual leadership as the ability of leaders to manage and guide the members to realize their personal expectations and then achieve the organizational goals by using the network media as the communication media under the influence of the network

${ }^{*}$ Corresponding author's e-mail: zhangyushi07@outlook.com 
intermediary factors. Due to the influence of many objective factors, we tend to work in this way. The work team can operate through the network, which can greatly reduce the conflict pressure brought by real-time work. Employees have higher quality subjective initiative. In this process, the intervention of artificial intelligence will greatly improve the work efficiency of the team. We assume that virtual leadership is an individual, that is, "virtual leadership", then he is a person with equal value in a virtual team. His characteristics and advantages lie in that he will not do complicated emotional thinking, but will judge the value according to the derivative of the established algorithm and finally choose the feasible path..

\subsection{The value of virtual leadership in Al environment}

There is no doubt that there are differences between human and machine learning, human learning autonomy and adaptability is strong, in unstructured and non fixed situations of decision-making and response and processing is much higher than the machine, otherwise the machine has more advantages. Kaifu Li believes that artificial intelligence will replace human resources to a large extent and bring impact to human resource management. Artificial intelligence has made a major breakthrough in deep learning[3]. In the future artificial intelligence environment, big data and massive computing are the core of mastering artificial intelligence technology. In this process, the management leadership of rational man can reach a tacit understanding with the intervention of artificial intelligence, which is reflected in the objective complementarity between human rationality and artificial intelligence. On the other hand, virtual leadership can realize its cross domain value.

\section{Application scope and significance of organizational virtual leadership}

\subsection{Application scope of organizational virtual leadership}

In the information age, great changes have taken place in the business environment, organizational structure and work style of the organization. Although some scholars have studied the leadership in the information age, most of them are from the perspective of chief technology officer (CTO), it director, and political and business leaders to study the ability of leaders in the process of organizational informatization construction. The current research is still in the exploratory stage, and there is no systematic theoretical system. The ability that managers need to lead employees to complete tasks through information technology media is an important part of information leadership, and the general managers in the organization should become the main body of information leadership.

\subsection{Application significance of artificial intelligence intervention in organization virtual leadership}

Out of interest in leadership, people have formed many different theories and explanations about leadership from practice and research. Some people try to integrate many leadership theories into a complete framework. The architecture to be introduced here focuses on the main variables that affect the effectiveness of leadership. We basically assume that the intervention of artificial intelligence in virtual leadership can be expressed as a basic formula:

$$
L=f(l, g m, s) * A(n \%)
$$

The meaning of this formula is that the leadership process of virtual leadership is a function of leaders, group members (or followers) and other situational variables under the (overall) moderate influence of artificial intelligence, and the network influence of virtual leadership is reflected in the percentage. And based on this, we form a model.

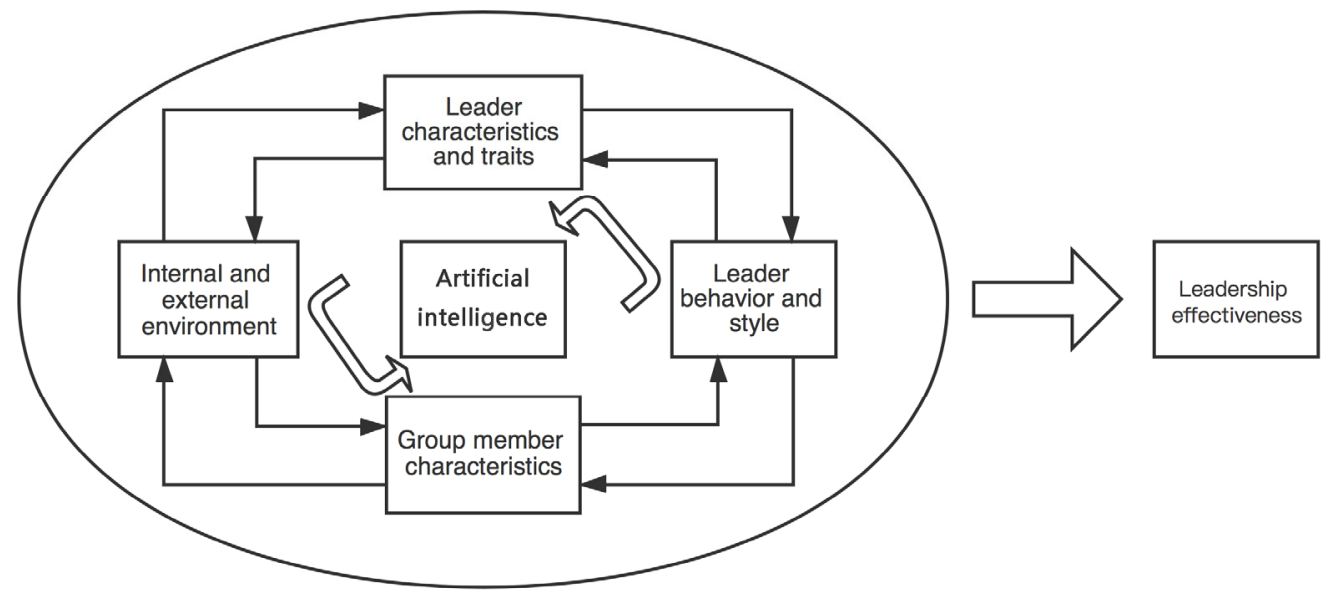

Fig 1. Virtual leadership model with AI intervention

\subsubsection{The virtual leadership of artificial intelligence can effectively improve the leadership ability and level}

The foothold of this part lies in the individual leader. Artificial intelligence is almost objective, which depends on whether the developer's original code is objective or not. We discuss it based on objectivity. Artificial intelligence helps to improve the ability of scientific 
decision-making. Scientific decision-making is a process of obtaining and processing the existing information. The rich data resources contained in the big data technology system can help the virtual leaders of artificial intelligence quickly obtain the data information related to decisionmaking in a short time, and make the decision-making basis change from a small amount of "sample data" to a large amount of "overall data", which is helpful to realize the scientific decision-making. This makes the leadership decision-making under the network platform a dynamic process of raising, analyzing and solving problems.

\subsubsection{The virtual leadership of artificial intelligence can effectively improve the decision-making standard of work}

The foothold of this part lies in the actual effect of the work. The virtual leadership of artificial intelligence in improving work efficiency is mainly manifested in four aspects: information advantage, efficiency advantage, competitive advantage and cost advantage. The virtual leadership provided by artificial intelligence can respond quickly and provide rapid support for project discussion due to its "own system" integrity In terms of competitive advantage and cost advantage, the virtual leadership provided by artificial intelligence can greatly improve the work efficiency of individual units, and then reduce the unit work cost.

\section{What are the ethical risks of $\mathrm{Al}$ intervention in virtual leadership?}

From the essence of thinking, the fundamental difference between artificial intelligence and automation is that artificial intelligence has self-consciousness and object consciousness. People should start with strengthening people's control and constructive participation to solve the challenges of AI ethics. Although the world is vigorously developing artificial intelligence, but the research of artificial intelligence ethics is relatively weak. However, the combination of human and machine capabilities will increase the ethical risks faced by human beings. On the surface, this kind of ethical risk is reflected in three kinds of dangerous deep thinking:

- The better artificial intelligence centered on human development develops, the more likely the best and brightest of us are to give up thinking

- No matter how much human experience a computer system absorbs, no matter how strong its ability to imitate human reasoning, we can not rely on an algorithm to maintain the emotional factors in leadership

- It may simply replicate human racial stereotypes and biased decisions. The deep ethical problems are privacy leakage, algorithm discrimination and subject infringement.

\subsection{Uncontrollable privacy disclosure}

Virtual leadership in the intelligent environment may have the supreme power, it can collect data, use scripts to track and record every move of the team. Data mining technology can find valuable information in a variety of seemingly unrelated data. However, once the information is collected by the data platform and stored in the cloud, it may cause a large area of privacy leakage once there is a protection vulnerability[4]. The wide application of cloud computing and data sharing technology aggravates the risk of privacy disclosure.

\subsection{Algorithm discrimination with obvious tendency}

Artificial intelligence system based on algorithm model and data learning human behavior data, and then make analysis and prediction. However, the training data may not truly reflect the objective reality, they may be interfered or polluted by some counter samples, making them make some wrong decisions[5]. Moreover, there may be hidden value preferences or customs that are not easy to be detected in these data. Racial discrimination or gender discrimination in a specific group may eventually be reflected in the data and inherited by machine learning.

\subsection{Violation of the power of artificial subject}

Hawking once warned that artificial intelligence is the real terminator of human beings, and the thorough development of artificial intelligence may lead to the extinction of human beings. Therefore, the evolution speed of strong artificial intelligence or super artificial intelligence is much faster than that of human beings, and the ultimate goal will be unpredictable. Therefore, the purpose of creating artificial agent must be positioned on enhancing human intelligence and serving human beings, rather than replacing human beings; in the process of creating artificial agent, human value judgment, moral requirements and intuition must be implanted into various artificial agents, so as to ensure that they are faithful to the original intention and mission of creating artificial agent in the application process.

\section{Conclusion}

The virtual leadership of artificial intelligence intervention organization must have the presence of ethics. Therefore, the purpose of creating artificial agent must be positioned on enhancing human intelligence and serving human beings, rather than replacing human beings; in the process of creating artificial agent, human value judgment, moral requirements and intuition must be implanted into various artificial agents, so as to ensure that they are faithful to the original intention and mission of creating artificial agent in the application process. 


\section{Acknowledgments}

This work was supported by Research on social governance mechanism based on network game theory (no.20191sljdwtkt-027)

\section{References}

1. Christian Graham,Harold Daniel. Fault Lines in Virtual Team Leadership and Team Performance in Undergraduate Virtual Team Short-Term Projects[J]. International Journal of e-Collaboration (IJeC),2021,17(1).

2. Mansour, N.|Rafeh, W. $\mid$ Afram, G. |Zaatari, G.. The Role of Self-Awareness, Augmented Artificial Intelligence and Enhanced Leadership Competencies in Developing Future Academic Physicians[J]. World Family Medicine Journal: Incorporating the Middle East Journal of Family Medicine,2019,17(10).

3. Demystifying Artificial Intelligence: A ForwardThinking Discussion with BlueBee Leadership on the Growing Clinical Role of Artificial Intelligence[J]. Clinical OMICs,2019,6(S1).

4. Human resource management in project-based organizations: revisiting the permanency assumption[J]. The International Journal of Human Resource Management,2021,32(1).

5. Yiou Huang. Research on the Talent Training Mode of Cooperation between Government, University and Enterprise of Human Resource Management Major in Application Oriented Universities[J]. Scientific Journal of Economics and Management Research,2021,3(1). 